\title{
REDIA: Designing a Repository for Agriculture and Food Research Data
}

\author{
Antonio Sánchez-Padial, Jorge García-Pérez \\ Instituto Nacional de Investigación y Tecnología Agraria y Alimentaria
}

\section{Keywords:}

Information systems design, data repository, research data, open data, open science, agriculture, food

\section{Speaker:}

Antonio Sánchez-Padial, antonio.sanchez@inia.es

\section{Bio:}

Antonio Sánchez-Padial is a Spanish Research Software Engineer. He has developed most of his career in several research centres applying his skills to domains such as environmental studies, groundwater research or humanities and social sciences communication. Since 2014 he works at Instituto Nacional de Investigación y Tecnología Agraria y Alimentaria (INIA) where he is part of the team developing a new research data management platform.

$\mathrm{He}$ is also a founder of the Processing Madrid community, where artists and developers meet every month for sharing their projects and experiences in creative coding. He has also volunteered as visualization designer for the artwork PRISM by artist Keiichi Matsuda, shown at Victoria \& Albert Museum during London Fashion Week 2012.

\begin{abstract}
:
INIA, the Spanish National Centre for Research and Technology in Agriculture and Food, is sited in Madrid and its nearby. It holds centres, departments and research groups in a wide spectrum of domains including forestry, animal health, animal breeding, environment, plant protection and others. But INIA is not only a research centre, in addition it's been in charge of Spanish national funding program for agriculture research until 2016. Furthermore it acts as a coordinator for the Spanish agricultural research network, made of several regional agriculture research centres across the country.
\end{abstract}


Accessing research data once projects are closed and after results are published becomes more difficult with time. According to Vines 2014, probability of accessing those data decreases up to $17 \%$ every year after publishing results. We don't have precise numbers for INIA though, but we know we suffer that risk extensively, with many junior researchers leaving the organization every year after getting their $\mathrm{PhD}$, and also senior staff leaving for retirement. Deploying an organization plan for research data management, supported by an information system, is a strategic issue for us.

Losing access to data is not the only threat we face. As most research organizations, we see how more and more funders and editors ask to publish research data with open licenses, either for accessing finantiation or publishing. Nowadays, there are a number of alternatives for publishing research data, but no one has zero inconveniences. Private repositories owner's goals can be opposite to those of a public research organization as we are. Public repositories like Zenodo - more about it below - are a good option, but then we would lack the opportunity to address the specific needs of agriculture and food research. Building our own repository looks like the best option, as far as we can engage and align with other organizations in our domain and avoid duplicating efforts, and as we manage to deal with long-tail data, because our institute holds a wide range of research groups and interests.

Data preservation and accomplishment with funders and publishers mandates are INIA's main goals for the data repository project. Moreover, we are adding the FAIR Guiding Principles, Wilkinson 2015, too. Findability, accessibility, interoperability and reusability have the same level of relevance than preservation or open data mandates. There is little sense in an open data platform that doesn't promote FAIR principles, it becomes a silo. Our view is a repository that serves as an exploration field for agricultural research, and a member of a linked data network delivering answers to a number of agricultural, nutrition and environmental complex queries launched both by researchers and public.

There are two things that make our researchers unwilling to collaborate in publishing and/or sharing their raw data. The first one is fear of data parasytes: researchers who will create new results with our original research data as source. Classic researches see those parasites as unfair competitors when they publish their results: they have no need to request competitive grants in order to get their things done, what makes them faster at delivering results. We consider the answers to this fear is in the hands of policymakers both with a stick, making compulsory to share raw research data in order to access fundings, and some carrots, promoting to share data by considering them research results in a similar level than article or book publishing. In some sense this is out of the scope of our project, though it can affect seriously its success. The platform will support this strategy issuing DOIs for every dataset published, and providing multilevel quoting both for datasets and their content.

The second problem that makes our researchers worry is that research data management may become another cumbersome clerical job, subtracting them time off of their 
research. This problem can be addressed from the technical side and/or from the local research policy (at the level of our organization). The goal here is to make as straightforward as possible the process for managing and publishing data. The platform will offer a couple of tools to our scientists. First, the Metadata Templating system, that lets researchers reuse most past of their metadata, and probably standardize them along departments and research groups. The second is the Default Research Data Management Plan, that users can choose for their projects and proposals when no other limitation applies.

For accomplishing our goal of avoiding to become a silo, and be a successful FAIR repository we have gathered a collection of regulations, partners and best-practices that we want to match with. We can classify them in three groups. The first one is the Spanish Law for Reuse of Information. This law sets conditions for what, when and how public information has to be published in a easy to reuse way. Scientific research is out of the scope of this law - our repository is not bound to it; however we are voluntarily committing to its reglaments, specially those concerning interoperability because we want our data to be linked or findable by public and researchers using other repositories under the scope of this law, such as environmental, social or weather repositories with data published by other Spanish administrations.

Research data management is the second domain we want our repository to fit with. EUDAT and the Research Data Alliance were the first knowledge sources we consulted to learn how to improve our interoperability, or what could be expected of our system. Studying their results and guidelines has been (is being!) very useful in order to solve (even before they happen) many issues, not only in the interoperability or semantic domains, but also in systems architecture and data management policies. MAREDATA is a research network created recently in Spain for sharing experiences and setting best practices in research data management, so it's another actor in this scope we will walk along with.

The third domain where our repository is expected to be able to work with others is agricultural data. The GODAN (Global Open Data for Agriculture and Nutrition) initiative, some working groups in the Research Data Alliance and FAO are some of the sources we take inspiration from.

INIA's infrastructures are far of being optimal. We have suffered bandwidth shortage for a long time, and even now that we are facing a new project to improve our physical communication infrastructure we are very concerned about working with big files up and down through our network. We are also worried about the increasing demand of storage that some disciplines (most in the end) require. Last but not least in our technical challenges list, accesing better computing performance is also related with the repository as research data will be both origin and result of those computing works. Moving data through our strangled network sounds like a pain we would like to avoid to our researchers. EUDAT's architecture (specially B2STAGE) has been very inspiring to solve these problems, even if we are not using it as is. 
From the very beginning, experts in research data management (Martínez Uribe from Fundación Juan March, Imma Subirats from FAO or Carlos Quirós from ILRI) recommended us to use a specific tool for data management such as Dataverse or CKAN instead of a generic content management system, a documentation tool like dSpace, or building a platform from scratch. So we runned a test comparing Dataverse and CKAN with our requirements. At the end of the test, we added ePrints to the experiment after we discovered many organizations used it too for research data. All the three tools fit very well our needs, Dataverse scored better in research data management aspects, and CKAN did its best in open data publishing. Both CKAN and ePrints seems to be better at customization, an important point because none of them three complied with all our requirements by default. CKAN was selected because it's written in Python, and it's easier to find developers and companies with experience in that language than in Perl, used in ePrints.

We expect to have a working repository next year. Meanwhile we have created a community in Zenodo, where our researchers can publish research data if they were requested so by publishers. Zenodo will support us in the future for transferring those datasets to our system, including DOIs. They also offered to keep that content as support repository. Our goal is to serve as national agriculture research data repository in the next future, with other organizations using our platform or making interoperable clones. We also are interested in working on international interoperability as soon as possible, and we would like to partner with another european actor in our domain, for sharing agriculture research data (at last metadata) in more detail than generic protocols allow. In the mid term we would like that the repository supports a data ecosystem of standards in agriculture research data formats growing out of the experience of many researchers sharing their datasets and accessing others'. We may expect those standards to become the backbone of more fine-grained interoperability with linked data.

Right now, we are creating the standards for managing, publishing and sharing research data at INIA: working on tutorials and documentation for Data Licenses, Data Intellectual Property and a Default Research Data Management Plan for our research projects.

\section{References}

Vines, T.H. et al, 2014. The Availability of Research Data Declines Rapidly with Article Age. Current Biology 24, 94-97. doi:10.1016/j.cub.2013.11.014

Wilkinson, Mark D. et al., 2016. The FAIR Guiding Principles for scientific data management and stewardship. Scientific Data 3, 160018. doi:10.1038/sdata.2016.18 